\title{
Article \\ Characterization of Genetically Modified Microorganisms Using Short- and Long-Read Whole-Genome Sequencing Reveals Contaminations of Related Origin in Multiple Commercial Food Enzyme Products
}

\author{
Jolien $D^{\prime}$ aes ${ }^{1,+}$, Marie-Alice Fraiture ${ }^{1,+} \mathbb{D}$, Bert Bogaerts ${ }^{1,2} \mathbb{D}$, Sigrid C. J. De Keersmaecker ${ }^{1}(\mathbb{D}$, \\ Nancy H. C. Roosens $1, \mp$ (iD) and Kevin Vanneste ${ }^{1, *, \ddagger}$ \\ 1 Transversal Activities in Applied Genomics (TAG), Department Expertise and Service Provision, Sciensano, \\ J. Wytsmanstraat 14, 1050 Brussels, Belgium; Jolien.Daes@sciensano.be (J.D.); \\ Marie-Alice.Fraiture@sciensano.be (M.-A.F.); Bert.Bogaerts@sciensano.be (B.B.); \\ Sigrid.DeKeersmaecker@sciensano.be (S.C.J.D.K.); Nancy.Roosens@sciensano.be (N.H.C.R.) \\ 2 Department of Plant Biotechnology and Bioinformatics, Ghent University, 9000 Ghent, Belgium \\ * Correspondence: kevin.vanneste@sciensano.be \\ + Equal first-author contribution. \\ $\ddagger$ Equal last-author contribution.
}

check for updates

Citation: D'aes, J.; Fraiture, M.-A.; Bogaerts, B.; De Keersmaecker, S.C.J.; Roosens, N.H.C.; Vanneste, K. Characterization of Genetically Modified Microorganisms Using Short- and Long-Read

Whole-Genome Sequencing Reveals Contaminations of Related Origin in Multiple Commercial Food Enzyme Products. Foods 2021, 10, 2637. https://doi.org/10.3390/ foods10112637

Academic Editor: Sílvia Bofill-Mas

Received: 8 October 2021

Accepted: 28 October 2021

Published: 30 October 2021

Publisher's Note: MDPI stays neutral with regard to jurisdictional claims in published maps and institutional affiliations.

Copyright: (c) 2021 by the authors. Licensee MDPI, Basel, Switzerland. This article is an open access article distributed under the terms and conditions of the Creative Commons Attribution (CC BY) license (https:/ / creativecommons.org/licenses/by/ $4.0 /)$.

\begin{abstract}
Despite their presence being unauthorized on the European market, contaminations with genetically modified (GM) microorganisms have repeatedly been reported in diverse commercial microbial fermentation produce types. Several of these contaminations are related to a GM Bacillus velezensis used to synthesize a food enzyme protease, for which genomic characterization remains currently incomplete, and it is unknown whether these contaminations have a common origin. In this study, GM B. velezensis isolates from multiple food enzyme products were characterized by shortand long-read whole-genome sequencing (WGS), demonstrating that they harbor a free recombinant pUB110-derived plasmid carrying antimicrobial resistance genes. Additionally, single-nucleotide polymorphism (SNP) and whole-genome based comparative analyses showed that the isolates likely originate from the same parental GM strain. This study highlights the added value of a hybrid WGS approach for accurate genomic characterization of GMM (e.g., genomic location of the transgenic construct), and of SNP-based phylogenomic analysis for source-tracking of GMM.
\end{abstract}

Keywords: genetically modified microorganism (GMM); Bacillus velezensis; food enzyme; wholegenome sequencing; hybrid genome assembly; SNP phylogenomic analysis

\section{Introduction}

Enzymes, additives and flavorings produced by microbial fermentation are widely used and indispensable for the food and feed industry. Genetically modified microorganisms (GMM) are frequently employed to increase microbial enzyme production efficiency and/or yield [1]. However, their presence is unauthorized in the final products commercialized in the European Union (EU) food and feed chain (EC/2003/1830). Moreover, since these GMM usually carry antimicrobial resistance (AMR) genes used as selection markers, the ingestion of such contaminated products has raised health concerns related to potential AMR horizontal gene transfer to pathogens and other gut microbiota.

Several unexpected contaminations of GMM carrying AMR genes, consisting of DNA as well as living GMM, were recently reported in microbial fermentation food and feed products commercialized on the EU market [2-7]. Control of potential GMM contaminations in microbial fermentation products is therefore crucial. In contrast to genetically modified organisms (GMO) directly intended for human and animal consumption, manufacturers of GMM-derived fermentation products do not need to provide GMO detection 
methods. To ensure food safety and traceability, as well as the consumer's freedom of choice, we have recently developed a GMM detection strategy using real-time PCR, a technology commonly mastered by enforcement laboratories performing GMO routine analysis. GMM presence is first screened for by targeting sequences frequently found in GMM, including three AMR genes ( $c a t$, aadD and tet-l, encoding chloramphenicol, kanamycin and tetracyclin resistance, respectively) and the pUB110 shuttle vector carrying aadD. Following a positive signal for at least one of these screening markers, the presence of specific GMM is investigated by targeting their unnatural associations [2-7].

Although such a qPCR strategy allows to prove the presence of a (specific) GMM in a given sample, further investigation is needed to obtain accurate risk assessment. More specifically, if a transgenic construct carrying AMR genes is harbored in the host on a free plasmid, the risk for horizontal transfer of AMR to other, potentially harmful, microorganisms may be greater than when the same transgenic construct is integrated into the microbial host genome. To perform in-depth genetic characterization, short-read whole-genome sequencing (WGS) has previously been used on a vitamin B2-producing GM Bacillus subtilis strain (RASFF2014.1249) [7,8], and the protease-producing GM B. velezensis that is the focus of this study (RASFF2019.3332) [2]. Moreover, the benefit of additionally using long-read WGS data in a hybrid assembly approach was successfully demonstrated for the vitamin B2-producing GM B. subtilis strain [9]. However, despite these successful case studies, the use of WGS for the characterization of GMM is currently still very limited.

Furthermore, no strategy is currently available to determine potential links between different contaminated products. Bacteria are genetically more flexible than plant or animal genomes. This can allow, for example, transformation of multiple parental strains with the same recombinant plasmid. Targeting an unnatural association on a free plasmid does not allow to differentiate between potentially different host strains, even from different species. Additionally, due to the variable possible outcomes of the recombination process leading to genomic integration of a construct, targeting the insertion of the construct in the genome does not provide a unique signature for a specific GMM, contrary to using an event-specific qPCR assay covering the unnatural association for GM plants. For source-tracking of bacterial outbreaks, e.g., identification of contaminated food leading to a food-borne outbreak, the current state-of-the-art approach is WGS of potential bacterial causal agents that were isolated from both the patients and the material, e.g., foodstuff, under suspicion, followed by phylogenetic analysis based on single-nucleotide polymorphisms (SNPs) [10,11]. A similar strategy might be used to investigate whether GMM contaminations originate from a common 'source', which in this case would pertain to a common parental GMM host strain used to manufacture different fermentation products.

Using WGS, this study reports an in-depth genomic characterization and phylogenomic comparison of 10 protease producing GM B. velezensis strains, carrying a transgenic construct derived from shuttle vector pUB110, that were isolated from four different commercial FE products. To unambiguously determine both the location and copy number of the transgenic construct, GM bacterial isolates were subjected to both short-read Illumina and long-read Oxford Nanopore Technology (ONT) sequencing to employ a de novo hybrid assembly strategy and additional in-depth bioinformatics investigation. We demonstrate that the GMM primarily carry the transgenic construct, with a single copy of the wild-type derived protease encoding gene, on a free high-copy pUB110-derived plasmid. Additionally, transient unstable integration of this transgenic construct into the chromosome can occur. Comparative phylogenomics analysis was performed to investigate the relationship between the GMM isolated from the different FE products, demonstrating that the obtained GMM were genetically almost identical, indicating that they likely originate from the same GMM strain and presumably manufacturer. 


\section{Materials and Methods}

\subsection{GMM Isolation from FE Products}

Four FE products in solid form were collected from the market (Table 1). One gram of each batch tested from each FE product was added to $250 \mathrm{~mL}$ of Brain-Heart Infusion broth (Sigma-Aldrich) in the presence of Kanamycin $(50 \mu \mathrm{g} / \mathrm{mL}$; Sigma-Aldrich) for incubation overnight at $30^{\circ} \mathrm{C}$. An amount of $100 \mu \mathrm{L}$ of the culture was plated on nutrient agar (SigmaAldrich) in the presence of Kanamycin (50 $\mathrm{\mu g} / \mathrm{mL}$; Sigma-Aldrich) for incubation overnight at $30^{\circ} \mathrm{C}$. Based on real-time PCR analysis (see Section 2.2), isolates of the GM B. velezensis producing protease were selected for genomic analysis. For each batch tested from each FE product, two isolates were selected, to investigate the presence of potentially different strains, as well as to serve as a back-up against potential loss of plasmids from the isolates.

Table 1. Overview of GMM isolates, and their associated commercial FE products and RASFF identifiers included in this study. Batch numbers denote different commercial FE product packages.

\begin{tabular}{|c|c|c|c|c|c|}
\hline $\begin{array}{l}\text { Commercial FE Product } \\
\text { (Supplier) }\end{array}$ & Associated RASFF & Labeled Enzymes & Application & Evaluated Batch & $\begin{array}{c}\text { Obtained GMM } \\
\text { Isolates }\end{array}$ \\
\hline $\begin{array}{c}\text { Alpha-amylase enzyme } \\
4 \mathrm{~g} \text { (Coobra) }\end{array}$ & RASFF2020.2577 & Alpha-amylase & Distillery & 1 & $\begin{array}{l}\text { Cob9-1 } \\
\text { Cob9-2 }\end{array}$ \\
\hline $\begin{array}{l}\text { Crystalmash (The } \\
\text { Alchemist's Pantry) }\end{array}$ & RASFF2019.3332 & $\begin{array}{l}\text { Alpha-amylase, } \\
\text { Protease, Cellulase, } \\
\text { Xylanase, } \\
\text { Beta-glucanase }\end{array}$ & Distillery, Brewing, Grain processing & 1 & $\begin{array}{l}\text { Crystal-1 } \\
\text { Crystal-2 }\end{array}$ \\
\hline \multirow{2}{*}{ Enzyme 4 g (Pilsner) } & \multirow{2}{*}{ RASFF2020.2582 } & \multirow{2}{*}{ Alpha-amylase } & \multirow{2}{*}{ Distillery, Brewing } & 1 & $\begin{array}{l}\text { Pilsner1-1 } \\
\text { Pilsner1-2 }\end{array}$ \\
\hline & & & & 2 & $\begin{array}{l}\text { Pilsner2-1 } \\
\text { Pilsner2-2 }\end{array}$ \\
\hline $\begin{array}{c}\text { Pureferm (The } \\
\text { Alchemist's Pantry) }\end{array}$ & RASFF2019.3332 & Neutral protease & $\begin{array}{c}\text { Beer and other cereal based beverages; } \\
\text { Bakery products and other cereal based } \\
\text { products }\end{array}$ & 1 & $\begin{array}{l}\text { Pure-1 } \\
\text { Pure-2 }\end{array}$ \\
\hline
\end{tabular}

\subsection{Real-Time PCR Colony Assays}

The selection of the bacterial isolates was determined using a real-time PCR marker (GMMprotease-R) specific to a GM B. velezensis producing protease developed and published previously (RASFF2019.3332) [2]. Each real-time PCR colony assay was performed in a standard $25 \mu \mathrm{L}$ reaction volume containing $1 \mathrm{X}$ TaqMan ${ }^{\circledR}$ PCR Mastermix (Diagenode, Liège, Belgium), $400 \mathrm{nM}$ of each primer (Eurogentec, Liège, Belgium) and $200 \mathrm{nM}$ of the probe (Eurogentec, Liège, Belgium). The real-time PCR program consisted of a single cycle of DNA polymerase activation for $10 \mathrm{~min}$ at $95^{\circ} \mathrm{C}$ followed by 45 amplification cycles of $15 \mathrm{~s}$ at $95^{\circ} \mathrm{C}$ (denaturing step) and $1 \mathrm{~min}$ at $60^{\circ} \mathrm{C}$ (annealing-extension step). All runs were performed on a CFX96 Touch Real-Time PCR Detection System (BioRad, Hercules, CA, USA). For each assay, a positive control (genomic DNA from the protease producing GM B. velezensis reported by [2] and an NTC (no template control) was included.

\subsection{DNA Extraction, Library Preparation and Whole Genome Sequencing}

DNA from GMM bacterial isolates was extracted using Quick-DNA ${ }^{\mathrm{TM}} \mathrm{HMW}$ MagBead Kit (Zymo Research, Irvine, CA, USA) according to the manufacturer's instructions and then visualized by capillary electrophoresis using the Tapestation 4200 device with the associated genomic DNA Screen Tape and reagents (Agilent, Santa Clara, CA, USA) (Figure S1). Each DNA concentration was measured by spectrophotometry using the Nanodrop ${ }^{\circledR} 2000$ (ThermoFisher, Dover, DE, USA) and each DNA purity was evaluated using the A260/A280 and A260/A230 ratios.

Plasmid DNA from the GM bacterial isolate Cob9-1 (Table 1) was extracted using the QIAprep Spin Miniprep Kit (QIAGEN, Hilden, Germany) according to manufacturer's instructions. For the plasmid DNA, only short-read sequencing was performed.

Short-read DNA libraries were prepared using the Nextera XT DNA library preparation kit (Illumina, San Diego, CA, USA) according to manufacturer's instructions. Sequencing was carried out on an Illumina MiSeq system with the V3 chemistry, obtaining $250 \mathrm{bp}$ 
paired-end reads, and aiming for a theoretical coverage of $60 \times$ per sample, based on the average Bacillus genome size of $\sim 4 \mathrm{Mbp}$.

Long-read DNA libraries were prepared using the ligation sequencing kit (SQKLSK109; Oxford Nanopore Technologies, Oxford, UK) and barcoding kit (EXP-NBD104; Oxford Nanopore Technologies, Oxford, UK) according to the manufacturer's instructions. Two groups of five DNA libraries individually barcoded were prepared and each group was loaded on an individual R9 MinION flow cell to be sequenced for $48 \mathrm{~h}$.

\subsection{Genome Assembly and Characterization}

Raw short reads were preprocessed with Trimmomatic 0.38 [12] with the following settings: ILLUMINACLIP:NexteraPE-PE.fa:2:30:10, LEADING:10, TRAILING:10, SLIDINGWINDOW:4:20, MINLEN:50. Quality of raw and preprocessed data was evaluated using FastQC 0.11.5 (www.bioinformatics.babraham.ac.uk/projects/fastqc, accessed on 14 August 2021)) with default settings.

Raw long reads were basecalled and demultiplexed with Guppy 4.2.3 in GPU mode. Guppy_barcoder was run with the_-trim_barcodes option to remove barcodes. Filtlong 0.2.0 (github.com/rrwick/Filtlong, accessed on 15 August 2021) was applied to raw fastq data to remove reads with an average quality score below 7 and read lengths below $1000 \mathrm{bp}$. Quality statistics on raw and filtered data were collected with NanoPlot 1.33.0 [13] with default settings.

For long read assembly, Canu 1.8 [14] was used, with the genome size set at $4.5 \mathrm{Mbp}$ and default settings. The resulting assembly was subjected to iterative short-read polishing with the Unicycler-polish module of Unicycler 0.4.7 [15], using default settings and the following dependencies: ALE v20180904 [16], Pilon 1.23 [17], Bowtie2 2.3.4.3 [18] and samtools 1.9 [19].

Hybrid assembly was carried out with Unicycler 0.4.7 and hybridSPAdes 3.13.0 [20]. The following Unicycler dependencies were employed: SPAdes, Pilon 1.23, Bowtie2 2.3.4.3, Racon 1.3.1 [21] and samtools 1.9. The Canu assembly was provided to Unicycler to be used instead of the default long read assembly that Unicycler produces internally, since we observed the final assembly being less fragmented using this approach. Unicycler uses this long read assembly in a hybrid strategy to improve the final assembly, which is based on both short and long reads. Otherwise, default settings were used. HybridSPAdes was run with the '-careful' argument, $\mathrm{k}$ set to '21, 33, 55, 77, 99, 127', and coverage cutoff set to 10.

The MiSeq data generated from the plasmid DNA extract was assembled with Unicycler in short-read mode, with default settings.

For subsequent whole genome comparison, a representative assembly was selected for each isolate, i.e., the Unicycler assembly when available, and the Canu assembly otherwise (Table S1).

Assembly statistics were obtained with Quast 5.0.2 [22], and assembly graph visualization with Bandage 0.8.1 [23]. Genome annotation was done with Prokka 1.11 [24], with default settings. Characterization of prophage sequences was conducted through the web interface of PHASTER [25]. Genotypic AMR detection was performed as described in Bogaerts et al. [26], with one modification, i.e., the National Database of Antibiotic Resistant Organisms (NDARO) (retrieved on 12 January 2021) was used instead of the ResFinder database.

\subsection{Follow-Up Analysis of Long Reads}

The workflow is represented as a flowchart in Figure S2. BLAST 2.7.1+ was run on raw long read datasets using pUB110 (Genbank: M19465.1) as query, increasing the maximum target sequences to 1 million to retain all possible hits. The resulting hits were filtered from the raw data with the filterbyname.sh script of bbtools 38.34 (Sourceforge.Net/Projects/ Bbmap, accessed on 14 August 2021), with default settings, except for the addition of 'include $=\mathrm{t}^{\prime}$ to retain hits matching the names instead of excluding them. Statistics on the filtered dataset were collected with NanoPlot. 
A subset of obtained BLAST hits, with read length of at least $6756 \mathrm{bp}$, to exclude sequences that are smaller than the pUB110-derived recombinant plasmid length, was visualized with the web interface of Kablammo [27]. Specific reads were manually selected for further analysis with web-based BLAST against the NCBI nucleotide collection database. This analysis yielded a read of $\sim 44 \mathrm{kbp}$ in length originating from sample Crystal-1, displaying a 2-copy chromosomal integration of the transgenic construct at the site of the wildtype protease encoding gene, which was used as reference for subsequent longread mapping.

A nested BLAST and filtering strategy was used, starting with a BLAST search of the raw long read datasets with either 2000 bp upstream or downstream of the chromosomal (wildtype) protease gene as queries. The hits were filtered as described above, and the filtered read dataset was subjected to a second round of BLAST, this time using pUB110 as query, after which hits were filtered again. The reads in the final read set were thus assumed to cover the putative site of chromosomal integration of the transgenic construct at the site of the wild-type protease gene. The read discovered during the analysis described in the previous paragraph was used as reference, to which all the other filtered reads were mapped with Minimap2 2.17 with the map-ont preset options [28]. Alignments were converted to BAM format, sorted and indexed with SAMtools 1.9, and visualized with Integrated Genomics Viewer 2.4.10 [29].

\subsection{SNP Phylogeny and SNP Typing}

To construct SNP-based phylogenies of the GMM isolates, all publicly available Illumina paired-end data of B. velezensis strains were collected via the ENA API (January 2021). The 42 retrieved datasets were combined with the Illumina paired-end data of the GM isolates, and a SNP matrix was obtained by running the CFSAN SNP pipeline [30] on processed unorphaned forward and reverse reads for each sample. The Unicycler hybrid assembly of isolate Pilsner1-2 was taken as reference since it contained the least fragmented assembly of all. The number of SNPs, without using any filters, was used to estimate the distance of the strains to the reference. Strains that showed more than 10,000 SNPs were excluded from further analysis, in accordance with the readme documentation of SnapperDB (github.com/phe-bioinformatics / snapperdb, accessed 15 August 2021). All GM strains of this study were retained and supplemented with two public datasets (Tables S4 and S6) using this criterion. The CFSAN pipeline was then rerun on the reduced sample set, with the same reference. The preserved (filtered) SNP matrix was used for evolutionary model selection through the web interface of SMS 1.8.4 [31]. Subsequently, a maximum-likelihood phylogenetic tree was constructed with PhyML 3.3.3:3.3.20170530+dfsg-2 [32] with the settings recommended by SMS: GTR substitution model, proportion of invariable sites fixed, 1 substitution rate category, and SPR tree topology search mode. One hundred bootstrap replicates were performed.

Additionally, SNP addresses of the reduced sample set were extracted with PHEnix 1.4.1 [33] and SnapperDB 1.0.6 [33] with the same reference genome, as described by Nouws et al. [34]. The 7-number SNP addresses provide an isolate level hierarchical clustering nomenclature, whereby isolates sharing an increasing amount of SNPs are increasingly more closely related. For example, for a group of isolates sharing only the first SNP address number, each isolate in the group is less than 250 SNPs away from at least one other isolate in the group. Likewise, the SNP thresholds are 100, 50, 25, 10, 5 and 0 for the second to last SNP address numbers, respectively.

Lastly, to obtain pairwise SNP distances for the GM isolates of this study, the CFSAN pipeline was run as described above, but solely including the 10 GM isolates of this study, with the same reference, and converted to a SNP distance matrix with snp-dists 0.7.0 (github.com/tseemann/snp-dists, accessed on 14 August 2021). 


\subsection{Whole Genome Alignment-Based Comparison}

For each isolate, the representative assembly obtained as described in Section 2.4 was employed. To facilitate interpretation, these assemblies were first manually pruned to retain only the largest contigs, accounting for at least $91.6 \%$ of the total assembly size in all cases (Table S2). This also implied that all contigs carrying the transgenic plasmid were removed from the assemblies. Multiple genome alignment was done with progressiveMauve 20150213 [35] with default settings, and included pruned annotated assemblies of the $10 \mathrm{GMM}$ isolates of this study, B. velezensis 10075, B. velezensis CBMB205 (Genbank: NZ_CP011937), which is the representative strain for this species in the RefSeq database, and a number of arbitrarily chosen $B$. velezensis strains, for which complete assemblies were publicly available (see Figure S14 for a complete list). The resulting alignments were visualized and investigated in Mauve viewer.

ProgressiveMauve 20150213 with the -seed-family option was also employed for multiple genome alignment of a putative plasmidic phage and a selection of known linear plasmidic phages, for which details are provided in Figure S6. Prior to the alignment, the MauveContigMover tool of Mauve 20150213 was employed to put all the sequences in the same orientation and facilitate their comparison.

\section{Results and Discussion}

\subsection{Isolation of Viable GM B. velezensis Producing Protease from FE Products, Long- and Short-Read WGS}

Previously, Fraiture et al. [2] described the genomic characterization of a viable $B$. velezensis GMM, isolated from a commercial FE product, more specifically a protease (i.e., 'Pureferm' in Table 1). Based on these findings, qPCR methods were developed that could subsequently be used to detect the presence of DNA from this unauthorized proteaseproducing GMM in other commercial FE products (Table 1) [6]. Among these FE products, in contrast to the Pureferm product labeled as protease, two were labeled as alpha-amylase and one was labeled as an enzyme mixture, among which were alpha-amylase and protease. From all these FE products, viable isolates of a protease-producing B. velezensis GMM could be obtained, which was reported at the EU level via the Rapid Alert System for Food and Feed (RASFF) notifications. Following confirmation by qPCR that they carried the transgenic protease construct, ten isolates from four different FE samples were selected for genomic characterization. An overview of the strains included in this study, with the FE products and batches from which they were isolated, is presented in Table 1.

A limitation of the former study of Fraiture et al. [2] was that the WGS data consisted of short-reads (Illumina) only, resulting in a relatively fragmented assembly. Although the presence of a GMM was firmly established, the generated short-read assembly did not allow to confidently determine the genomic location and copy number of the transgenic construct. Over the past few years, third-generation sequencing technologies, such as Oxford Nanopore Technologies (ONT), have become widely accessible, and increasingly performant, facilitating the generation of much longer reads, albeit at a lower accuracy, compared to reads from the dominant second-generation sequencing platform Illumina. Consequently, hybrid assembly methods have been developed that allow to exploit the strengths of both short- and long-read sequencing technologies, i.e., the low sequencing error rate of the Illumina technology to obtain very accurate assemblies, and the capacity of long reads to bridge and resolve repetitive regions in the genome [36]. A (more) complete, accurate genome assembly could allow to unambiguously establish the location of the transgenic construct in a GMM, i.e., whether it is plasmidic or genomic, and its copy number. A recent study by Berbers et al. [9] highlighted the added value of such a 'hybrid' approach for genomic characterization of GMM. Hence, we chose to sequence genomic DNA of isolates from the protease-producing GM B. velezensis using the Illumina and ONT technologies, in order to determine the location and copy number of the transgenic construct, and determine the relationship between the different FE products contaminated with this GMM. 
The two MinION flow cells, each containing five samples, delivered 5,387,509 reads with a read length N50 of $7646 \mathrm{bp}$ and median read quality of 12.1 and 7,211,191 reads with a read length N50 of $2603 \mathrm{bp}$ and median read quality of 11.8, respectively. After demultiplexing, the number of reads per sample ranged from 101,521 to 2,422,322, and the read length N50 per sample varied between 1951 and 12,809 bp. Read quality was very similar for the different samples. Per-sample metrics are provided in Table S3.

Raw short-read input varied from 461,014 to 729,454 per sample (Table S3) and was generally of very high quality. In the GC distribution plot of the FastQC report (Figure S3) of all isolates, the main peak was located at $45-46 \% \mathrm{GC}$, corresponding to the expected value of $45.6-47 \% \mathrm{GC}$ reported for complete chromosome assemblies of $B$. velezensis in the RefSeq database (date retrieved: 03/2021). Besides this main peak, a conspicuous broad secondary peak was present at $28-37 \%$ GC, in line with the expected value of $35.7 \%$ for plasmid pUB110 (Genbank: M19465.1). This pronounced secondary peak was congruent with the presence of pUB110 as a free high-copy number plasmid, and/or the integration of a substantial number of pUB110 copies into the chromosome.

\subsection{Genetic Characterization of GMM}

The overview of the genetic characterization of the protease-producing $B$. velezensis GMM provided in this section was obtained by integrating results from different assembly approaches, complemented with additional in-depth investigation of raw long read data. A more detailed description of each of these analyses and their contribution to the full characterization is available in the supplementary information.

\subsubsection{Characterization of GMM Host Strain}

Figure 1 depicts graphs of the Unicycler, Canu, and hybridSPAdes assemblies of sample Pilsner1-2, which was chosen as representative isolate because it presented the least fragmented hybrid assembly. Assembly graphs for the other isolates were similar for each assembly method, and are shown in Figure S4. The main assembly stats for all assemblies are presented in Table S1.

With respect to the chromosome, the publicly available $B$. velezensis genome that was most similar to that of the GMM isolates of this study based on web-based blastn, was that of $B$. velezensis 10075, a strain isolated from food lobster sauce in China. This strain was therefore also included in the comparative genomic analysis (see Section 3.3.2). Next to the chromosome, all but one of the protease GMM isolates carried an extrachromosomal natural element, which is most likely a plasmidic prophage. No AMR genes were detected on the chromosome or putative plasmidic prophage of the GMM isolates. In all the isolate assemblies, the only AMR genes present were associated with the transgenic construct (see Section 3.2.2).

\subsubsection{The Transgenic Modification Is Present as an Episomal High-Copy Plasmid}

Based on the integration of the assembly results with the additional analyses, the hybrid assembly via Unicycler was able to most accurately reflect the ground truth of the protease GMM isolates, i.e., with the transgenic construct on a high-copy free plasmid of $6756 \mathrm{bp}$ in length (Figure 1A) (Supp. text 1.1). Supporting evidence for this genetic make-up was provided by short-read sequencing and assembly of a plasmid extract, details of which are provided in the supplementary information, and by the presence of a distinct peak at 6.6-6.8 kbp visible in the read length histogram of raw pUB110-matching long-reads, corresponding to the length of the recombinant plasmid (Figure S8) (Supp. text 2.1). The high-copy nature of the plasmid in Bacillus spp. is described in the literature [37], and is supported by the high read-depth for the plasmid-covering reads, as compared to the read-depth observed for genome-covering reads for all the isolates (results not shown). 


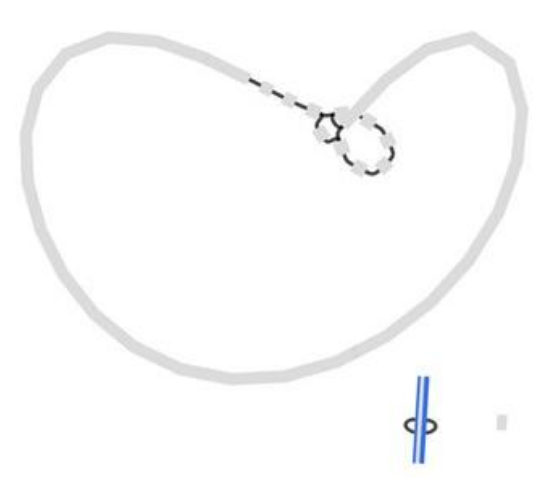

A

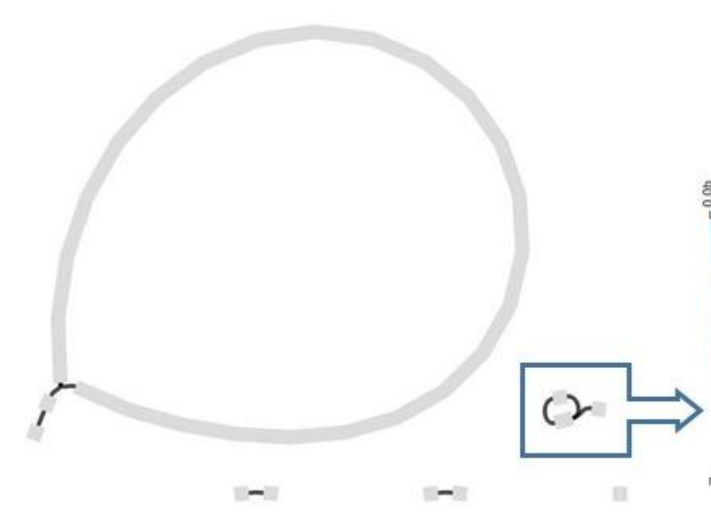

C

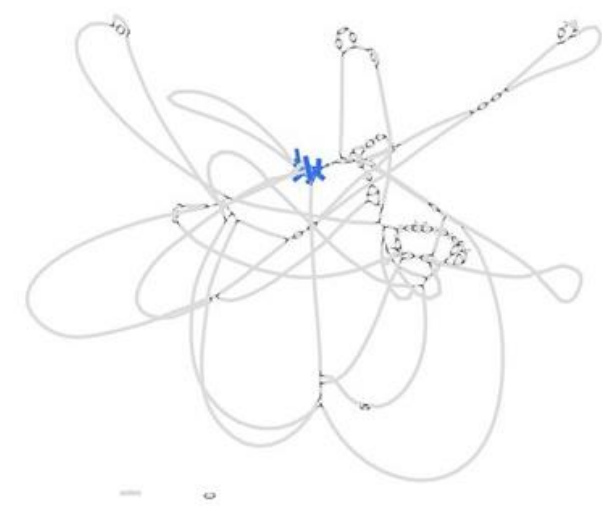

B

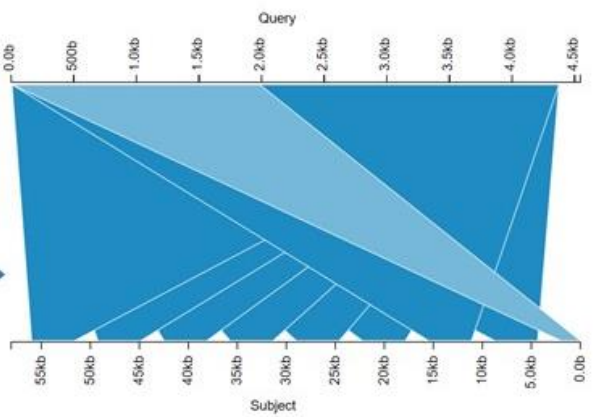

D

Figure 1. Assembly graphs of Unicycler, hybridSPAdes, and Canu assemblies of GM isolate Pilsner12. (A): Unicycler assembly of Pilsner1-2, with the pUB110 sequence on the plasmid contig in the assembly highlighted in blue, and the contig representing the putative plasmidic prophage highlighted in green. (B): HybridSPAdes assembly, with the pUB110 sequence inside the chromosome scaffold highlighted in blue, and the contig representing the putative plasmidic prophage highlighted in green. (C): Canu assembly with the three contigs that display recombinant plasmid concatemers framed in blue. (D): Kablammo visualization of the largest of the three contigs carrying concatemers of the Canu assembly. The top ruler represents the plasmid pUB110, and the bottom ruler represents the contig, containing 8 complete copies of the recombinant plasmid.

However, because Unicycler failed to run to completion for 7 out of 10 samples, an alternative hybrid assembly tool, hybridSPAdes, was also run on the samples. HybridSPAdes yielded very fragmented assemblies, showing integration of the transgenic construct into the chromosome, contrary to the Unicycler assemblies (Figure 1B) (Supp. text 1.2). Therefore, Canu, a long-read only assembly method, was also executed, which supported the presence of the transgenic construct as an extrachromosomal structure, but not of the integration that was apparent in the HybridSPAdes assemblies. Noteworthy, contrary to the single-copy circular plasmid revealed in the Unicycler assemblies, all Canu assemblies exhibited one or more contigs carrying concatemers, i.e., multiple contiguous head-to-tail copies, of the recombinant plasmid (Figure 1C,D) (Supp. text 1.3).

Figure 2 depicts the genetic structure of the high-copy recombinant plasmid, derived from the pUB110 shuttle plasmid. Part of the pUB110 sequence was deleted, and replaced by a recombinant insert (Figure S5) encoding a protease gene that is $100 \%$ identical to the natural protease gene in the chromosome of the B. velezensis host strain. The transgenic 
construct harbored two AMR genes: aadD, conferring kanamycin and neomycin resistance, and ble, conferring bleomycin resistance. All elements required for normal replication [37] had remained intact, indicating that the vector was designed to be episomal rather than integrative [1] (Supp. text 2.2).

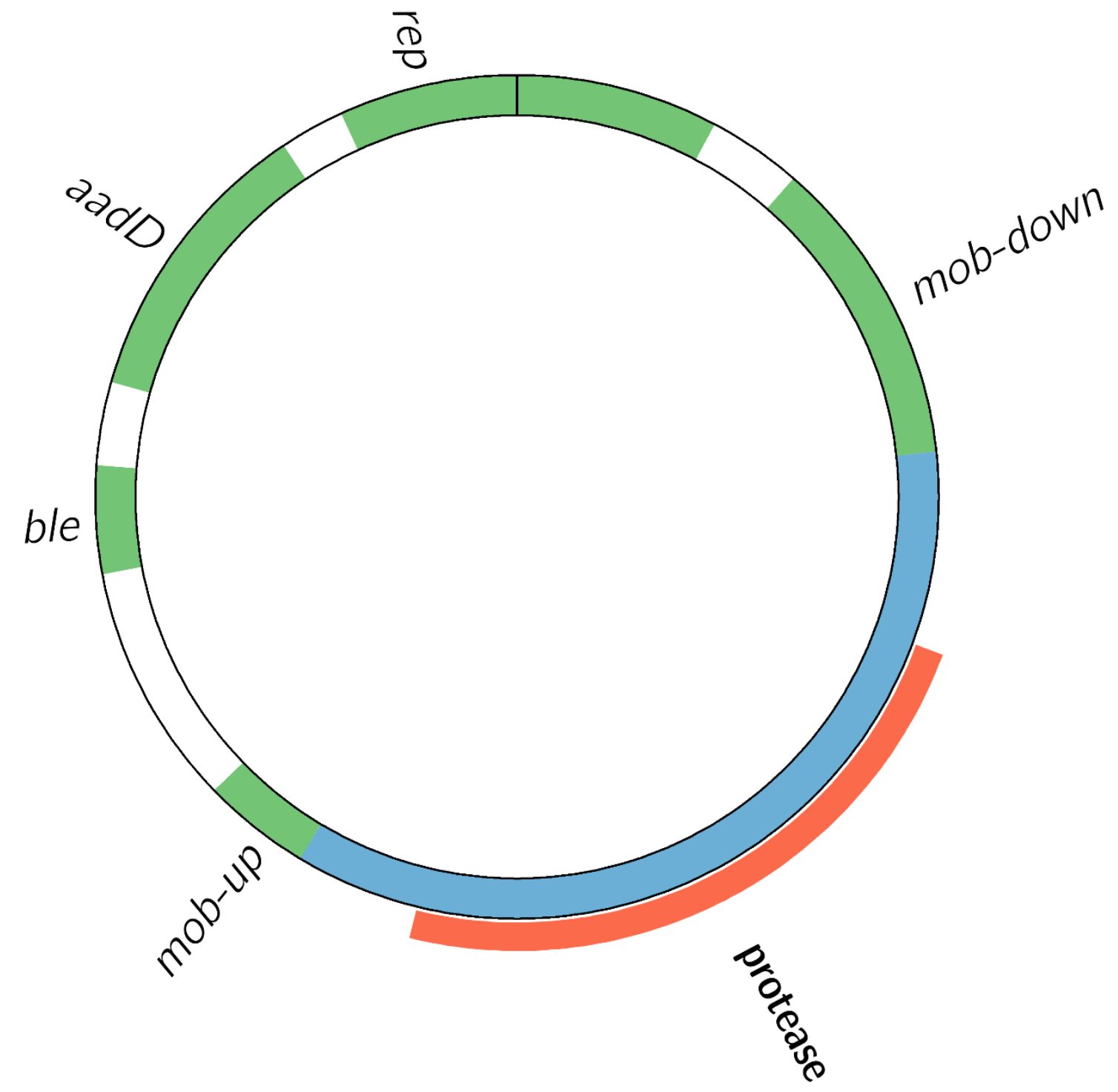

Figure 2. Map of the recombinant 6756 bp plasmid carried by the GM isolates. aadD: kanamycin and neomycin resistance gene, ble: bleomycin resistance gene, rep: replicase, mob: mobilization protein. A part of the mob gene is absent compared to the original pUB110 vector, and is replaced with a recombinant insert of $2385 \mathrm{bp}$ in length, highlighted in blue, encompassing a complete protease encoding gene, highlighted in orange, which is also present in the chromosome of the isolates, as well as in the chromosome of B. velezensis 10075. Figure created with Circos 0.69-6 [38].

3.2.3. The Transgenic Plasmid Shows Sporadic, Unstable Integration into the Chromosome and Its Replication Is Disturbed, Leading to Accumulation of Linear Plasmid Concatemers

Our investigation revealed two additional findings of interest. First, we found evidence that despite the transgenic construct being primarily carried on a free high-copy plasmid, unstable transient chromosomal integration did appear to occur to some limited extent. This was supported by a limited number of raw long reads, observed for all isolates, that exhibited one to two contiguous copies of the plasmid integrated at the site of the natural chromosomal copy of the protease encoding gene (Figure 3). The fact that this vector can sporadically integrate into the host genome is an additional reason for concern, 
as this presumably homology-driven integration event may similarly occur in related naturally occurring strains that have taken up the vector by horizontal gene transfer.

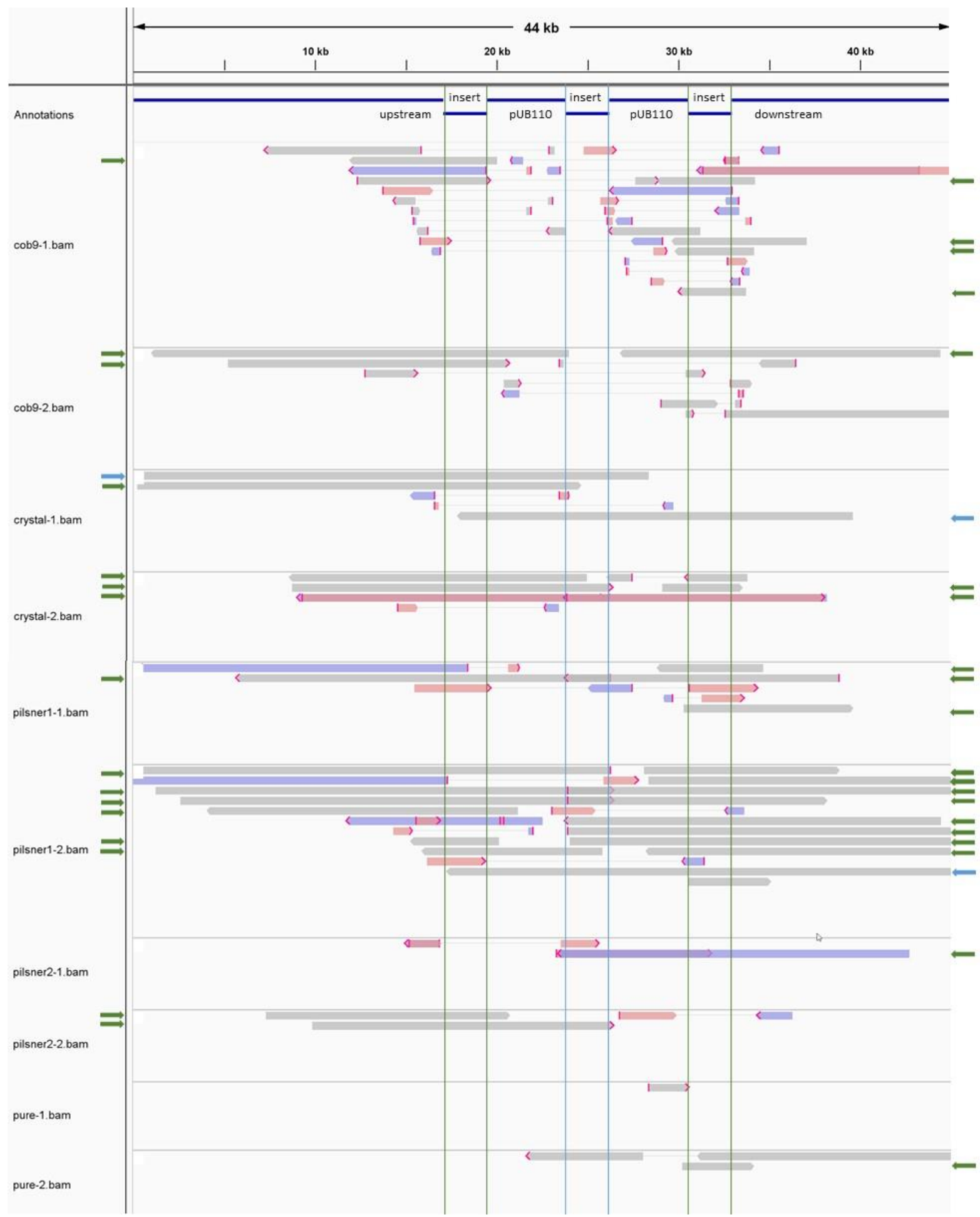

Figure 3. Alignment to reference read of $\sim 44 \mathrm{~kb}$, originating from isolate Crystal-1, displaying a two-copy integration of the recombinant plasmid. The alignment shows all raw long reads of all isolates (as labeled in the first column 'Annotations') that match pUB110 as well as chromosomal sequence upstream or downstream of the sequence used as insert in the transgenic construct, which carries the wild-type protease (hereafter referred to as 'insert'). These reads are thus expected to cover the junction of chromosomal integration of the recombinant plasmid. If a supplementary alignment was reported, its connection to the primary alignment is shown via a thin grey line. The primary, or representative, alignment is the highest 
scoring alignment for a particular read. If the read does not align in its entirety, parts of the read sequence may be clipped, i.e., removed from the alignment. In some cases, (mostly) non-overlapping parts of the read may align at different positions to the reference. In that case, the non-primary alignment(s) is/are referred to as supplementary alignment(s). Primary and supplementary alignments that are inverted compared to each other are marked in red (forward alignment compared to the reference) and blue (reverse complement alignment). Clipped regions are hidden, and, if they are $>30 \mathrm{bp}$, flagged with a red mark. For clarity, secondary alignments (same part of the read from the primary alignment, but aligning at another location to the reference), mismatched bases (variants) and short indels are hidden. Green vertical lines mark the edges of the first and third copy of the insert, which is present three times in the reference, as a consequence of the double plasmid integration event that is represented by the reference. The blue vertical lines mark the end of the first, and beginning of the second, integrated pUB110 copy in the reference read, with in between another (third) copy of the insert. If a continuous alignment extends beyond two adjacent green lines, it supports a chromosomal integration of at least one pUB110 copy. These reads are marked with a green arrow. If the alignment extends beyond both blue lines, in addition to two adjacent green lines, it supports the presence of (at least) two contiguous integrated pUB110 copies. These reads are flagged with a blue arrow. Figure created with IGV.

Secondly, we observed for all isolates an abundance of raw long-reads displaying so-called plasmid concatemers, representing linear high molecular-weight head-to-tail copies of the recombinant pUB110-derived plasmid. As stated above, one or more contigs displaying these concatemers were present in all of the long-read (Canu) assemblies, while they did not show up in the hybrid (Unicycler) assemblies. The most likely explanation for this concatemer-related phenomenon is disturbed plasmid replication in the cells, since previous studies have reported that pUB110-replication can be disrupted under certain conditions, e.g., insertion of foreign DNA into pUB110, leading to accumulation of highmolecular-weight head-to-tail plasmid multimers in e.g., B. subtilis [39] (Supp. text 2.3).

Overall, our results highlight that correct assembly of a GMM, even with longread data, can pose specific challenges, hence requiring a particularly careful approach including multiple additional ad hoc analyses, instead of relying on the output of one particular assembler.

\subsection{Comparative Analysis and Source Tracing}

To determine the genomic relationship between the isolates from different FE products, and assess whether they potentially originate from a common source, two approaches were used to perform an in-depth comparative analysis.

\subsubsection{SNP Phylogenetic Analysis and Typing Indicate the Isolates Share a Common Source}

SNP phylogenetic analysis and SNP typing were combined to infer the phylogenomic context of the isolates, and quantify their differences. Based on an explorative screening analysis that included all available $B$. velezensis strains for which Illumina paired-end data was publicly available (Tables S4 and S5), only samples that differed by fewer than 10,000 SNPs from the reference isolate Pilsner1-2 were included in the SNP-based phylogeny and SNP typing, retaining only two public datasets in total. Although approximately 338 $B$. velezensis genome assemblies were publicly available in NCBI (date accessed March 2021), paired-end Illumina data were available for only 42 strains.

The resulting SNP phylogenetic tree and accompanying SNP addresses are shown in Figure 4 (CFSAN pipeline metrics in Table S6). All 10 isolates clustered together monophyletically, and were at least 250 SNPs away from the two other strains in the tree. The GM isolates shared always at least the first four digits of their SNP addresses, i.e., they were always within at least $25 \mathrm{SNPs}$ of one other isolate. Isolates originating from the same FE product clustered together, as expected. Moreover, Crystal and Pure isolates, which originate from the same supplier (see Table 1), also clustered together. This underpins the sensitivity of this method, and its potential for source-tracking of unauthorized GMM. Pilsner2-1 and Pilsner2-2 shared the same SNP address, i.e., no SNPs were found between both isolates. The same was true for Crystal-1 and Crystal-2. Pilsner1-1 and Pilsner1-2 shared the first six digits of their SNP address, implying that they differed by fewer than 
five SNPs. Inspection of their pairwise SNP distances (Table S7) showed that they actually differed by only one SNP. For Pure-1 vs. Pure-2, and Cob9-1 vs. Cob9-2, which shared only the first four digits of their SNP address, the difference was more notable, i.e., 16 and 10 SNPs difference, respectively (Table S7).

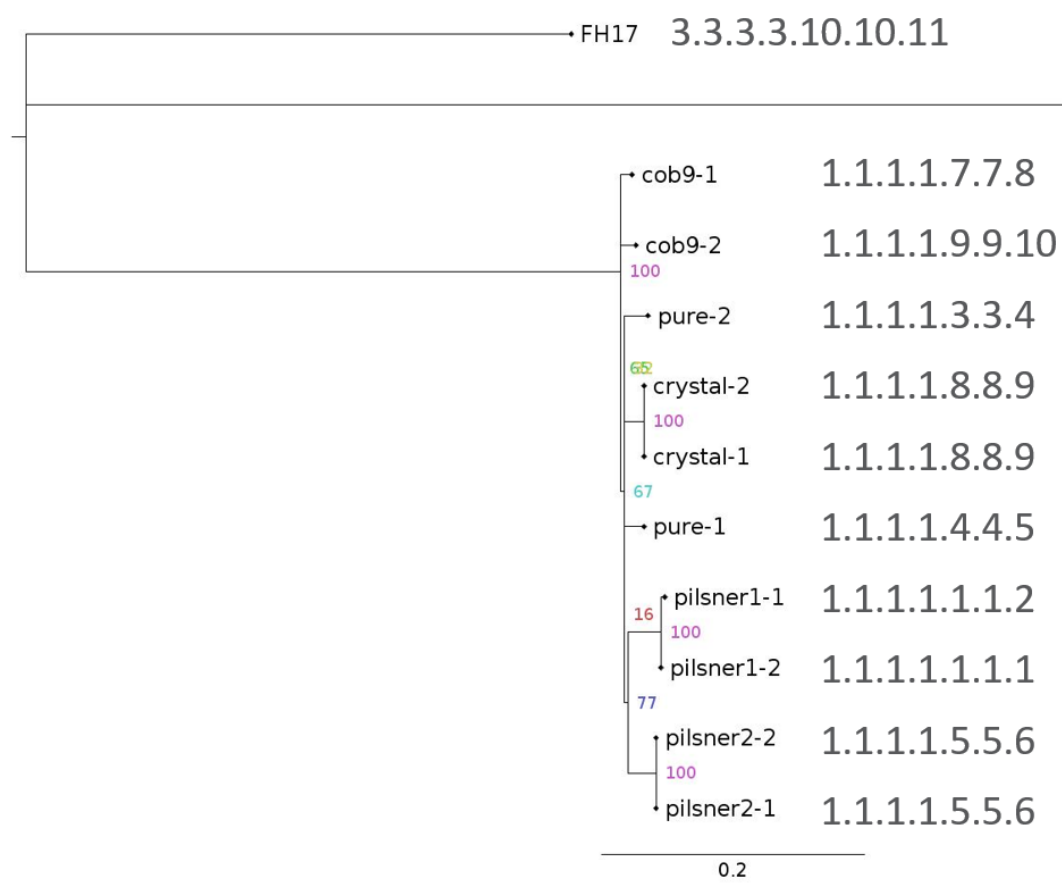

Figure 4. SNP-based phylogenetic tree, visualized with FigTree (github.com/rambaut/figtree, 29 October 2021), with associated SNP addresses. Both for SNP phylogeny and SNP typing, the Unicycler assembly of isolate Pilsner1-2 was used as reference. The scale is expressed as average substitutions per site in the SNP matrix. Node values represent bootstrap support values.

Recently, Pightling et al. [40] proposed a set of criteria to evaluate whether bacterial isolates share a common source, based on SNP phylogenies. The study focused on pathogenic bacteria and tracing of outbreak sources, but the general framework can equally well be applied to GMM, thus providing a useful and objective guideline. The criteria are threefold: (1) isolates cluster together monophyletically, which was the case here as the GM isolates were clearly delineated from the two background samples; (2) bootstrap support for the monophyletic cluster should be $>0.89$, and was $100 \%$ in this case; and (3) the isolates differ from each other by at most a few tens of SNPs, and in this case all GM isolates belonged to the same $25 \mathrm{SNP}$-cluster, with the largest pairwise distance between any two isolates being 21 SNPs (Table S7). Since all three criteria were fulfilled, this strongly supports that the isolates derive from the same parental strain used to manufacture the different GM fermentation products.

\subsubsection{Whole Genome Comparison Supports Results from SNP-Based Analysis}

A multiple genome alignment was performed to evaluate potential genomic rearrangements between isolates that could be missed by short read-based SNP analysis because of the short-read lengths. Overall, this analysis confirmed that the isolates displayed very high mutual sequence conservation, and that $B$. velezensis 10075 was highly related to the GMM isolates (Figure S10). This is illustrated by the presence of a putative prophage in the isolates, and in strain 10075, which was clearly absent from all other included B. velezensis strains (Figure S14). Although this region was practically identical in the 10 isolates, it showed marked sequence divergence compared to the homologous region in strain 10075, indicating a certain degree of evolutionary distance between 10075 and the GM isolates. 
Inspection of the genomic region around the chromosomal location of the protease encoding gene in the isolates and in strain 10075, indicated that this region was highly conserved in all 10 isolates compared to 10075, and no signs of recombination or presence of elements/genes that are not present in 10075 were detected, supporting the absence of genetic modification of the host genome within this region (Figure S11).

At certain sites, indications of rearrangements were however found between the isolates. Phage characterization of the representative Unicycler assembly of sample Pilsner12 (Table S8), and cross-comparison with the multiple genome alignment of the isolates (Figure S10) revealed that prophages appeared to be abundantly present, accounting for $\sim 416 \mathrm{kbp}$ in total, or $\sim 9.5 \%$, of the draft genome of isolate Pilsner1-2. All apparent intracontig break points coincided with prophage sequences, and most, but not all, contig boundaries were also found to occur at prophage sites, thus the apparent rearrangements likely represented misassembly artefacts. Alternatively, these could represent authentic rearrangement events, due to very recent prophage proliferation activity, or a combination of both. Nevertheless, in one specific case, clear signs of a large-scale rearrangement were found, i.e., in the Crystal isolates (Figures S12 and S13), where exactly the same locally collinear block (LCB) of $\sim 116 \mathrm{kbp}$, not associated with a prophage, was found to be rearranged compared to all other isolates and strain 10075.

\section{Conclusions}

In this study, the presence of viable GMM, carrying AMR genes, was observed in four commercial FE products, which was reported at the EU level via the Rapid Alert System for Food and Feed (RASFF) notifications (Table 1). By combining a variety of bioinformatics approaches using short-read and/or long-read data, backed up with experimental evidence, we were able to thoroughly characterize $10 \mathrm{GM}$ bacterial isolates, obtained from the different commercial FE products.

We could confirm that the three GM isolates for which a Unicycler assembly could be obtained, carry a recombinant plasmid, derived from pUB110, with an insertion containing the wild-type protease encoding gene. The presence of a peak at $6.6-6.8 \mathrm{kbp}$ in the read length histograms of all the isolates strongly supports that this plasmid is present in the other GM isolates as well. Furthermore, the recombinant plasmid is partly present in the form of linear plasmid multimers, probably due to a disturbance of the normal plasmid replication mechanism, and it is likely only transiently integrated into the chromosome. This study highlights the added value of a hybrid approach via WGS, since neither shortread nor long-read sequencing methods solely were able to correctly resolve the relatively complex genomic constitution of the GM isolates.

These findings raise serious food safety and public health concerns, which are emphasized by the fact that the AMR genes are harbored on a free plasmid, increasing the risk for spreading of AMR via horizontal gene transfer. They also highlight the need for thorough genetic characterization of GMM since the location, i.e., chromosomal or plasmidic, of the transgenic construct and associated AMR genes, is important information to consider for safety evaluation.

Furthermore, we could conclude that the GMM isolates from different FE products are genetically almost identical, and that they most probably originate from the same parental GMM strain. In particular, SNP-phylogenetic analysis based on short-read mapping was able to resolve the phylogenetic relationship between the isolates. To our knowledge, this study is the first to demonstrate the potential of such an approach for source-tracking of GMM. The outcome of the phylogenetic analysis was confirmed by whole-genome alignment-based comparison, while additionally demonstrating misassemblies or rearrangements that were caused by prophage sequences, and also the existence of one largescale rearrangement in the isolates obtained from one FE, which would have been left unnoticed otherwise. 
Supplementary Materials: The following are available online at https://www.mdpi.com/article/10 .3390 / foods10112637/s1, Supplementary text: Detailed explanation concerning elucidation of genetic make-up of GMM, Figure S1: Visualization of DNA extracted from isolates of the protease-producing GM B. velezensis strain, Figure S2: Workflow for analysis of raw long reads, as described in Material and methods section 2.5, Figure S3: Per sequence GC content of raw forward short reads from isolate Cob9-1 (output of FastQC), Figure S4: Assembly graphs, created with Bandage, for all successful assemblies, Figure S5: Alignment of the native pUB110 vector to the recombinant pUB110-derived plasmid from the GM isolates (created with Kablammo), Figure S6: Multiple genome alignment of known and putative linear extrachromosomal prophages from different Bacillus strains (alignment conducted with ProgressiveMauve, visualization in Mauve alignment viewer), Figure S7: Intact plasmid DNA from GM bacterial isolate Cob9-1, visualized by capillary electrophoresis using the Tapestation 4200 device, Figure S8: Read length profile of Cob9-1 raw long read data filtered to retain only reads matching pUB110 sequence, Figure S9: Top 25 hits of raw long reads of sample Pilsner1-2, BLAST analyzed using pUB110 as query, visualized with Kablammo, Figure S10: Multiple genome alignment, visualized with Mauve viewer, of the $10 \mathrm{GM}$ isolates and reference strain $B$. velezensis 10075, Figure S11: Whole genome comparison of 10 isolates and B. velezensis 10075, zoomed in on area around chromosomal protease encoding gene, Figure S12: Mauve alignment view of B. velezensis 10075 (uppermost track) and 10 GM isolates, showing a number of locally collinear blocks (LCBs), which represent regions of continuous sequence conservation among the different genomes, Figure S13: Mauve alignment view of the same multiple genome alignment as presented in Figure S12, from another vantage point, Figure S14: Mauve viewer snapshot of multiple genome alignment of, from top to bottom: B. velezensis strains Bac57 (NZ_CP033054), CBMB205 (NZ_CP011937), CGMCC11640 (NZ_CP026610), DKU_NT_04 (NZ_CP026533), DSYZ (NZ_CP030150), Lzh-a42 (NZ_CP025308), SRCM103616 (NZ_CP035410), W1 (NZ_CP028375),YAU B9601-2 (NZ_017061), 10075, and the GM isolates; Pilsner1-2 (designated as reference in the viewer), Pilsner1-1, Pilsner2-1, Pilsner2-2, Cob9-1, Cob9-2, Crystal-1, Crystal-2, Pure-1, Pure-2, Table S1: Metrics of Unicycler1, hybridSPAdes, and Canu assemblies for the $10 \mathrm{GM}$ isolates, Table S2: Overview of assemblies used as representative for each sample, and stats of pruned assemblies used in multiple genome alignment, Table S3: Key metrics for Illumina and ONT raw data of this study, Table S4: B. velezensis strains included in the SNP phylogeny analysis, Table S5: Metrics of CFSAN pipeline run on GM isolates under study, together with all B. velezensis strains for which paired-end Illumina data was publicly available (January '21), with the Unicycler assembly of isolate Pilsner1-2 as reference, Table S6: Metrics of CFSAN pipeline of selected sample set used for final SNP phylogeny, Table S7: SNP distance matrix with pairwise distances for the 10 GM isolates, Table S8: Summary of prophage analysis of complete Unicycler assembly of sample Pilsner1-2 with PHASTER.

Author Contributions: J.D.: Investigation, Formal analysis, Data curation, Methodology, Validation, Software, Writing—original draft preparation, Writing—review and editing, Visualization; M.-A.F.: Conceptualization, Investigation, Formal analysis, Data curation, Methodology, Validation, Resources, Writing-original draft preparation, Writing—review and editing; B.B.: Methodology, Resources, Software, Writing-review and editing; S.C.J.D.K.: Methodology, Resources, Writing-review and editing; N.H.C.R.: Conceptualization, Funding acquisition, Project administration, Supervision, Methodology, Writing-review and editing; K.V.: Conceptualization, Resources, Project administration, Supervision, Methodology, Writing-review and editing. All authors have read and agreed to the published version of the manuscript.

Funding: This research received no external funding.

Institutional Review Board Statement: Not applicable.

Informed Consent Statement: Not applicable.

Data Availability Statement: Raw data and assemblies were deposited in the European Nucleotide Archive under study accession number PRJEB44065.

Acknowledgments: This research was funded by the Transversal activities in Applied Genomics Service from Sciensano (Belgium). We thank the technicians of the Transversal Activities in Applied Genomics Service for performing the Illumina next-generation sequencing runs.

Conflicts of Interest: The authors declare no conflict of interest. 


\section{References}

1. Deckers, M.; Deforce, D.; Fraiture, M.A.; Roosens, N.H.C. Genetically modified micro-organisms for industrial food enzyme production: An overview. Foods 2020, 9, 326. [CrossRef] [PubMed]

2. Fraiture, M.-A.; Bogaerts, B.; Winand, R.; Deckers, M.; Papazova, N.; Vanneste, K.; de Keersmaecker, S.C.J.; Roosens, N.H.C. Identification of an unauthorized genetically modified bacteria in food enzyme through whole-genome sequencing. Sci. Rep. 2020, 10, 7094. [CrossRef] [PubMed]

3. Fraiture, M.-A.; Deckers, M.; Papazova, N.; Roosens, N.H.C. Detection strategy targeting a chloramphenicol resistance gene from genetically modified bacteria in food and feed products. Food Control. 2020, 108, 106873. [CrossRef]

4. Fraiture, M.-A.; Deckers, M.; Papazova, N.; Roosens, N.H.C. Strategy to detect genetically modified bacteria carrying tetracycline resistance gene in fermentation products. Food Anal. Methods 2020, 13, 1929-1937. [CrossRef]

5. Fraiture, M.-A.; Marchesi, U.; Verginelli, D.; Papazova, N.; Roosens, H.C. Development of a real-time PCR method targeting an unauthorized genetically modified microorganism producing alpha-amylase. Food Anal. Methods 2021, 14, 2211-2220. [CrossRef]

6. Fraiture, M.-A.; Papazova, N.; Roosens, N.H.C. DNA walking strategy to identify unauthorized genetically modified bacteria in microbial fermentation products. Int. J. Food Microbiol. 2021, 337, 108913. [CrossRef]

7. Paracchini, V.; Petrillo, M.; Reiting, R.; Angers-Loustau, A.; Wahler, D.; Stolz, A.; Schönig, B.; Matthies, A.; Bendiek, J.; Meinel, D.M.; et al. Molecular characterization of an unauthorized genetically modified Bacillus subtilis production strain identified in a vitamin B2 feed additive. Food Chem. 2017, 230, 681-689. [CrossRef]

8. Barbau-Piednoir, E.; de Keersmaecker, S.C.J.; Wuyts, V.; Gau, C.; Pirovano, W.; Costessi, A.; Philipp, P.; Roosens, N.H. Genome sequence of EU-unauthorized genetically modified Bacillus subtilis strain 2014-3557 overproducing riboflavin, isolated from a vitamin B2 80\% feed additive. Genome Announcements 2016, 3, e00214-15. [CrossRef]

9. Berbers, B.; Saltykova, A.; Garcia-Graells, C.; Philipp, P.; Arella, F.; Marchal, K.; Winand, R.; Vanneste, K.; Roosens, N.H.C.; de Keersmaecker, S.C.J. Combining short and long read sequencing to characterize antimicrobial resistance genes on plasmids applied to an unauthorized genetically modified Bacillus. Sci. Rep. 2020, 10, 4310. [CrossRef]

10. Nouws, S.; Bogaerts, B.; Verhaegen, B.; Denayer, S.; Crombé, F.; de Rauw, K.; Piérard, D.; Marchal, K.; Vanneste, K.; Roosens, N.H.C.; et al. The benefits of whole genome sequencing for foodborne outbreak investigation from the perspective of a national reference laboratory in a smaller country. Foods 2020, 9, 1030. [CrossRef]

11. Sekse, C.; Holst-Jensen, A.; Dobrindt, U.; Johannessen, G.S.; Li, W.; Spilsberg, B.; Shi, J. High throughput sequencing for detection of foodborne pathogens. Front. Microbiol. 2017, 8, 2029. [CrossRef] [PubMed]

12. Bolger, A.M.; Lohse, M.; Usadel, B. Trimmomatic: A flexible trimmer for Illumina sequence data. Bioinformatics 2014, 30, 2114-2120. [CrossRef]

13. De Coster, W.; D’Hert, S.; Schultz, D.T.; Cruts, M.; van Broeckhoven, C. NanoPack: Visualizing and processing long-read sequencing data. Bioinformatics 2018, 34, 2666-2669. [CrossRef] [PubMed]

14. Koren, S.; Walenz, B.P.; Berlin, K.; Miller, J.R.; Bergman, N.H.; Phillippy, A.M. Canu: Scalable and accurate long-read assembly via adaptive K-mer weighting and repeat separation. Genome Res. 2017, 27, 722-736. [CrossRef]

15. Wick, R.R.; Judd, L.M.; Gorrie, C.L.; Holt, K.E. Unicycler: Resolving bacterial genome assemblies from short and long sequencing reads. PLoS Comput. Biol. 2017, 13, e1005595. [CrossRef]

16. Clark, S.C.; Egan, R.; Frazier, P.I.; Wang, Z. ALE: A generic assembly likelihood evaluation framework for assessing the accuracy of genome and metagenome assemblies. Bioinformatics 2013, 29, 435-443. [CrossRef]

17. Walker, B.J.; Abeel, T.; Shea, T.; Priest, M.; Abouelliel, A.; Sakthikumar, S.; Cuomo, C.A.; Zeng, Q.; Wortman, J.; Young, S.K.; et al. Pilon: An integrated tool for comprehensive microbial variant detection and genome assembly improvement. PLoS ONE 2014, 9, e112963. [CrossRef] [PubMed]

18. Langmead, B.; Salzberg, S. Fast gapped-read alignment with Bowtie2. Nat. Methods 2012, 9, 358-359. [CrossRef]

19. Li, H.; Handsaker, B.; Wysoker, A.; Fennell, T.; Ruan, J.; Homer, N.; Marth, G.; Abecasis, G.; Durbin, R. The Sequence Alignment/Map format and SAMtools. Bioinformatics 2009, 25, 2078-2079. [CrossRef]

20. Bankevich, A.; Nurk, S.; Antipov, D.; Gurevich, A.A.; Dvorkin, M.; Kulikov, A.S.; Lesin, V.M.; Nikolenko, S.I.; Pham, S.; Prjibelski, A.D.; et al. SPAdes: A new genome assembly algorithm and its applications to single-cell sequencing. J. Comput. Biol. 2012, 49, 455-477. [CrossRef]

21. Vaser, R.; Sović, I.; Nagarajan, N.; Šikić, M. Fast and accurate de novo genome assembly from long uncorrected reads. Genome Res. 2017, 27, 737-746. [CrossRef]

22. Gurevich, A.; Saveliev, V.; Vyahhi, N.; Tesler, G. QUAST: Quality assessment tool for genome assemblies. Bioinformatics 2013, 29, 1072-1075. [CrossRef]

23. Wick, R.R.; Schultz, M.B.; Zobel, J.; Holt, K.E. Bandage: Interactive visualization of de novo genome assemblies. Bioinformatics 2015, 31, 3350-3352. [CrossRef]

24. Seemann, T. Prokka: Rapid prokaryotic genome annotation. Bioinformatics 2014, 30, 2068-2069. [CrossRef]

25. Arndt, D.; Grant, J.R.; Marcu, A.; Sajed, T.; Pon, A.; Liang, Y.; Wishart, D.S. PHASTER: A better, faster version of the PHAST phage search tool. Nucleic Acids Res. 2016, 44, 16-21. [CrossRef]

26. Bogaerts, B.; Nouws, S.; Verhaegen, B.; Denayer, S.; van Braekel, J.; Winand, R.; Fu, Q.; Crombé, F.; Piérard, D.; Marchal, K.; et al. Validation strategy of a bioinformatics whole genome sequencing workflow for Shiga toxin-producing Escherichia coli using a reference collection extensively characterized with conventional methods. Microb. Genom. 2021, 7, 000531. [CrossRef] 
27. Wintersinger, J.A.; Wasmuth, J.D. Kablammo: An interactive, web-based BLAST results visualizer. Bioinformatics 2015, 31, 1305-1306. [CrossRef] [PubMed]

28. Li, H. Minimap2: Pairwise alignment for nucleotide sequences. Bioinformatics 2018, 34, 3094-3100. [CrossRef]

29. Robinson, J.T.; Thorvaldsdóttir, H.; Winckler, W.; Guttman, M.; Lander, E.S.; Getz, G.; Mesirov, J.P. Integrative genomics viewer. Nat. Biotechnol. 2011, 29, 24-26. [CrossRef] [PubMed]

30. Davis, S.; Pettengill, J.B.; Luo, Y.; Payne, J.; Shpuntoff, A.; Rand, H.; Strain, E. CFSAN SNP pipeline: An automated method for constructing snp matrices from next-generation sequence data. PeerJ Comput. Sci. 2015, 1, e20. [CrossRef]

31. Lefort, V.; Longueville, J.E.; Gascuel, O. SMS: Smart Model Selection in PhyML. Mol. Biol. Evol. 2017, 34, 2422-2424. [CrossRef]

32. Guindon, S.; Dufayard, J.F.; Lefort, V.; Anisimova, M.; Hordijk, W.; Gascuel, O. New algorithms and methods to estimate maximum-likelihood phylogenies: Assessing the performance of PhyML 3.0. Syst. Biol. 2010, 59, 307-321. [CrossRef] [PubMed]

33. Dallman, T.; Ashton, P.; Schafer, U.; Jironkin, A.; Painset, A.; Shaaban, S.; Hartman, H.; Myers, R.; Underwood, A.; Jenkins, C.; et al. SnapperDB: A database solution for routine sequencing analysis of bacterial isolates. Bioinformatics 2018, 34, 3028-3029. [CrossRef]

34. Nouws, S.; Bogaerts, B.; Verhaegen, B.; Denayer, S.; Piérard, D.; Marchal, K.; Roosens, N.H.C.; Vanneste, K.; de Keersmaecker, S.C.J. Impact of DNA extraction on whole genome sequencing analysis for characterization and relatedness of Shiga toxin-producing Escherichia coli isolates. Sci. Rep. 2020, 10, 14649. [CrossRef]

35. Darling, A.E.; Mau, B.; Perna, N.T. Progressivemauve: Multiple genome alignment with gene gain, loss and rearrangement. PLoS ONE 2010, 5, e11147. [CrossRef] [PubMed]

36. Chen, Z.; Erickson, D.L.; Meng, J. Benchmarking hybrid assembly approaches for genomic analyses of bacterial pathogens using Illumina and Oxford Nanopore sequencing. BMC Genom. 2020, 21, 631. [CrossRef]

37. Viret, J.F.; Alonso, J.C. A DNA sequence outside the pUB110 minimal replicon is required for normal replication in Bacillus subtilis. Nucleic Acids Res. 1988, 16, 4389-4406. [CrossRef] [PubMed]

38. Krzywinski, M.; Schein, J.; Birol, I.; Connors, J.; Gascoyne, R.; Horsman, D.; Jones, S.J.; Marra, M.A. Circos: An information aesthetic for comparative genomics. Genome Research 2009, 19, 1639-1645. [CrossRef]

39. Gruss, A.; Ehrlich, S.D. Insertion of foreign DNA into plasmids from gram-positive bacteria induces formation of high-molecularweight plasmid multimers. J. Bacteriol. 1988, 170, 1183-1190. [CrossRef] [PubMed]

40. Pightling, A.W.; Pettengill, J.B.; Luo, Y.; Baugher, J.D.; Rand, H.; Strain, E. Interpreting whole-genome sequence analyses of foodborne bacteria for regulatory applications and outbreak investigations. Front. Microbiol. 2018, 9, 1482. [CrossRef] [PubMed] 